\title{
FATORES ASSOCIADOS À DESISTÊNCIA DE IDOSAS EM UM PRO- GRAMA DE ATIVIDADE FÍSICA: UM ESTUDO RETROSPECTIVO
}

\author{
Enaiane Cristina Menezes \\ Universidade do Estado de Santa Catarina, Florianópolis, Santa Catarina, Brasil. \\ Inês Amanda Streit \\ Universidade Federal do Amazonas, Manaus, Amazonas, Brasil \\ Eduardo Capeletto \\ Universidade do Estado de Santa Catarina, Florianópolis, Santa Catarina, Brasil. \\ Marina Ribovski \\ Universidade do Estado de Santa Catarina, Florianópolis, Santa Catarina, Brasil. \\ Artur Rodrigues Fortunato \\ Universidade do Estado de Santa Catarina, Florianópolis, Santa Catarina, Brasil. \\ Giovana Zarpellon Mazo \\ Universidade do Estado de Santa Catarina, Florianópolis, Santa Catarina, Brasil.
}

\begin{abstract}
Resumo
O objetivo foi verificar os fatores associados à desistência de idosas de um programa de atividades físicas. A amostra foi composta por 267 idosas participantes e desistentes de um programa de atividade física de extensão universitária. Para a coleta de dados foi utilizada a ficha com dados do programa. As idosas desistentes do programa $(n=108)$ apresentaram média de idade de 69,4 (dp=5,99) anos. Na análise bruta, as variáveis, estado civil e condições de saúde influenciaram na prática de atividade física. A doença cardíaca, depressão, estado civil e escolaridade foram associadas ao desfecho (desistência). Conclui-se que condições sociodemográficas, doença cardíaca e depressão são fatores associados à desistência do programa de atividade física.
\end{abstract}

Palavras-chave: Envelhecimento. Atividade Motora. Depressão. Insuficiência Cardíaca.

\section{FACTORS ASSOCIATED WITH THE WITHDRAWAL OF ELDERLY WOMEN IN A PHYSICAL ACTIVITY PROGRAM: A RETROSPECTIVE STUDY}

\begin{abstract}
The objective was to verify the factors associated with the withdrawal of the elderly from a physical activity program. The sample consisted of 267 older women who were withdrawn from a physical activity program of extension university. For the data collection, a data sheet with program data was used. The women who quit the program $(n=108)$ had a mean age of $69.4(\mathrm{SD}=5.99)$ years. In the crude analysis, the variables, marital status, and health conditions influenced the practice of physical activity. Heart disease, depression, marital status, and schooling were associated with outcome (withdrawal). We conclude that sociodemographic
\end{abstract}


conditions, heart disease, and depression are factors associated with the withdrawal of the physical activity program.

Keywords: Aging. Motor Activity. Depression. Heart Failure.

\title{
FACTORES ASOCIADOS A LA DESISTENCIA DE ANCIANAS EN UN PROGRA- MA DE ACTIVIDAD FÍSICA: UN ESTUDIO RETROSPECTIVO
}

\begin{abstract}
Resumen
El objetivo fue verificarlos factores asociados a la desistencia de ancianas de un programa de actividades físicas. La muestra fue compuesta por 267 ancianas participantes y desistentes de un programa de actividad física de extensión universitaria. Para la recolección de datos se utilizó la ficha con datos del programa. Las ancianas desistentes del programa $(n=108)$ presentaron un promedio de edad de 69,4 (dp=5,99) años. En el análisis bruta, las variables, estado civil y condiciones de salud influenciaron en la práctica de actividad física. La enfermedad cardíaca, depresión, estado civil y escolaridad fueron asociadas al desenlace (desistencia). Se ha observado que las condiciones sociodemográficas, la enfermedad del corazón y la depresión, son factores asociados con la retirada del programa de actividad física.
\end{abstract}

Palabras clave: Envejecimiento. Actividad Motora. Depresión. Insuficiencia Cardíaca.

\section{Introdução}

A saúde do idoso tem se tornado cada vez mais relevante devido ao aumento exponencial do envelhecimento na população, inclusive no Brasil (IBGE, 2013). Comparando dados de 2004 , em que, $9,7 \%$ da população era representada por idosos, no ano de 2014 , o percentual de pessoas com mais de 60 anos no país foi para 13,7\% (IBGE, 2015).

Considera-se que a atividade física influencia na diminuição da incapacidade do indivíduo e dos fatores de riscos para o desenvolvimento de doenças crônico-degenerativas, além de proporcionar inúmeros benefícios associados à sua prática (NELSON et al., 2007; WALSTON et al., 2006). No entanto, a inatividade física tem sido relatada como fator determinante para os gastos com saúde pública, impactando principalmente no número de internações hospitalares. Cerca de $15 \%$ dos custos das internações do Sistema Único de Saúde (SUS) foram atribuíveis à inatividade física (BIELEMANN et al., 2015).

Evidências na literatura mostram que a busca do idoso pela atividade física é motivada pela preocupação com a própria saúde, os relacionamentos interpessoais, o acompanhamento de familiares (MOURA; DE SOUZA, 2015). Além disso, a permanência desses idosos em programas de atividade física, também se dá pela convivência em grupo, pela saúde e melhora da qualidade de vida (LOPES et al., 2014; SALIN et al., 2014). Por outro lado, podem ser observados alguns fatores que dificultam esta prática pelo idoso, entre elas estão, o aparecimento de limitações físicas, a própria idade avançada, o acometimento por doença, a viuvez e a influência negativa do meio ambiente (KRUG; MAZO; LOPES, 2015; LOPES et al., 2016).

Observa-se que dentre as evidências supracitadas, a permanência em programas de atividades físicas ou a dificuldade para adesão aos mesmos são investigadas por meio da percepção subjetiva do idoso. Porém, a prática de atividade física é influenciada por determinantes sociais, demográficos, ambientais e individuais (BAUMAN et al., 2012), o que acarreta à necessidade de investigar variáveis clínicas. É evidenciado na literatura que a atividade física é fator de proteção para o desenvolvimento de determinadas doenças (YUSUF et al., 2004; 
SOFI et al., 2008; LEE et al., 2012; MIKKELSEN et al., 2010), mas, ainda é necessário investigar se as pessoas acometidas por esses problemas permanecem ativas fisicamente.

Dessa forma, compreender os fatores que levam à desistência da participação em programas de atividade física por idosas, pode contribuir para um melhor planejamento de programas a fim de motivá-las e envolvê-las na prática de atividade física. Sendo assim, o objetivo do presente estudo foi verificar os fatores associados à desistência de mulheres idosas de programas de atividades físicas.

\section{Método}

\section{Tipo de estudo}

O presente estudo é caracterizado como observacional analítico e retrospectivo (BLO$\mathrm{CH}$; COUTINHO, 2009), pois pretende verificar os fatores relacionados com a desistência das idosas de um programa universitário de atividade física. Neste tipo de estudo, a exposição (fatores) e o desfecho (desistência) são avaliadas após já terem ocorrido, através de relato ou registros.

\section{População e Amostra}

A população do estudo foi composta por idosas matriculadas nos projetos de atividades físicas do programa de extensão Grupo de Estudo da Terceira Idade (GETI), do Centro de Ciências da Saúde e do Esporte (CEFID) da Universidade do Estado de Santa Catarina (UDESC), nos anos de 2013, 2014, 2015 e 2016. Em cada ano foram matriculadas 300 idosas, aproximadamente. A matrícula nos projetos ocorreu, geralmente, no mês de março de cada ano. Os projetos de atividades físicas desenvolvidos foram: Hidroginástica, Natação, Ginástica, Musculação, Pilates, Dança e Caminhada.

Para definir a amostra do estudo, as idosas foram divididas em dois grupos: para o primeiro grupo (desistentes), teve-se como critério idosas que participaram do programa no ano de 2013 e não retornaram para a realização da matrícula nos anos de 2014e/ou 2015e/ou 2016. Para o segundo grupo (permanentes), adotou-se como critério estar matriculada nos anos de 2013 a 2016. Deste modo, participaram do estudo 267 mulheres idosas, sendo que 159 eram do grupo das que permaneceram e 108 as que desistiram do programa. As idosas que não realizaram a matrícula nos projetos durante o período investigado devido ao falecimento, foram excluídas do estudo.

\section{Instrumentos e Coleta de Dados}

Para este estudo, as informações foram retiradas do banco de dados do programa de extensão GETI/CEFID/UDESC referentes aos anos analisados - 2013, 2014, 2015 e 2016, após autorização deste pela coordenação do programa. As informações obtidas das idosas eram referentes aos dados sociodemográficos, condição atual de saúde e dificuldade para prática de atividade física habitual. Para o grupo de idosas permanentes foram utilizados os dados correspondentes ao ano de 2016. Já para ao grupo de idosas desistentes, foram utilizados os dados referentes ao último ano de participação no programa (2013 e/ou 2014 e/ou 2015 e/ou 2016). 


\section{Tratamento estatístico}

Os dados foram armazenados e analisados no programa estatístico SPSS versão 20.0. A análise descritiva dos dados foi realizada por meio de medidas de posição e dispersão (média e desvio padrão) e frequência relativa e absoluta para as variáveis categóricas. Todas as variáveis foram associadas com o desfecho por meio do teste Qui-Quadrado de Pearson. A análise dos fatores associados foi feita pela análise de regressão logística binária, sendo incluídas na análise bruta as variáveis que apresentaram associação no teste Qui-Quadrado com pvalor $<0,200$. A entrada das variáveis do modelo ajustado foi feita pelo método stepwise forward. O ajuste do modelo foi avaliado pelo teste de Hosmer-Lemeshow.

\section{Aspectos éticos}

O estudo foi aprovado pelo Comitê de Ética Envolvendo Seres Humanos da Universidade do Estado de Santa Catarina sob o número de protocolo, 185/2007 e 052406/2015.Para a realização dessa pesquisa, foram cumpridos os princípios éticos de acordo com a resolução 466/12 do Conselho Nacional de Saúde.

\section{Resultados}

Observa-se na Tabela 1 que as idosas permanentes $(n=159)$ no programa de atividades físicas do GETI apresentaram média de idade de 70,0 (DP=6,93) anos. Destas, 44,7\% eram casadas e 30,9\% eram viúvas, 30,9\% completaram o ensino médio e 19,1\% apresentaram ensino superior, e $25,7 \%$ recebiam de 2 a 3 salários mínimos. As idosas desistentes do programa $(\mathrm{n}=108)$ apresentaram média de idade de $69,4(\mathrm{DP}=5,99)$ anos. Destas $26,7 \%$ das idosas eram viúvas e $26,7 \%$ eram casadas, $30 \%$ completaram o ensino superior e $28 \%$ completaram o ensino médio, e $28,1 \%$ recebiam mais de seis salários mínimos. Na análise bruta, as variáveis estado civil, estado de saúde atual dificulta a prática de atividade física, doença cardíaca, depressão e problemas de visão foram associadas ao desfecho.

Tabela 1 - Análise descritiva e bruta dos fatores associados a desistência das idosas do programa de atividades físicas $(n=267)$

\begin{tabular}{|c|c|c|c|c|}
\hline Variável & $\begin{array}{c}\text { Permanentes } \\
(\mathbf{n}=159) \\
\text { n }(\%)\end{array}$ & $\begin{array}{c}\text { Desistentes } \\
(\mathbf{n}=108) \\
\text { n }(\%)\end{array}$ & $\begin{array}{c}\text { OR } \\
(\text { IC 95\% })\end{array}$ & Valor $\mathbf{p}$ \\
\hline \multicolumn{5}{|l|}{ Idade } \\
\hline 60-69 anos & $83(53,9)$ & $55(50,9)$ & 1 & \multirow{3}{*}{0,444} \\
\hline 70-79 anos & $54(35,1)$ & $43(41,7)$ & $1,20(0,71-2,03)$ & \\
\hline 80 anos ou mais & $17(11,0)$ & $05(4,9)$ & $0,44(0,15-1,27)$ & \\
\hline \multicolumn{5}{|l|}{ Estado civil } \\
\hline Com companheiro & $68(44,7)$ & $27(26,7)$ & 1 & \multirow{2}{*}{$0,004 *$} \\
\hline Sem companheiro & $84(55,3)$ & $74(73,3)$ & $2,22(1,29-3,82)$ & \\
\hline \multicolumn{5}{|l|}{ Com quem mora } \\
\hline Com alguém & $100(67,1)$ & $75(75,0)$ & 1 & \multirow{2}{*}{0,183} \\
\hline Sozinho & $49(32,9)$ & $25(25,0)$ & $0,68(0,39-1,20)$ & \\
\hline \multicolumn{5}{|l|}{ Escolaridade } \\
\hline Até 8 anos & $60(39,5)$ & $30(30,0)$ & 1 & \multirow{2}{*}{0,126} \\
\hline 8 anos ou mais & $92(60,5)$ & $70(70,0)$ & $1,52(0,89-2,60)$ & \\
\hline \multicolumn{5}{|l|}{ Renda } \\
\hline 1 a 2 SM & $41(27,0)$ & $20(31,3)$ & 1 & 0,581 \\
\hline
\end{tabular}




\begin{tabular}{|c|c|c|c|c|}
\hline 2 a 4 SM & $64(42,1)$ & $16(25,0)$ & $0,51(0,24-1,10)$ & \\
\hline 4 ou mais $\mathrm{SM}$ & $47(30,9)$ & $28(43,8)$ & $1,22(0,60-2,48)$ & \\
\hline \multicolumn{5}{|c|}{ Dificuldade para AF } \\
\hline Não & $115(77,7)$ & $63(64,3)$ & 1 & \multirow{2}{*}{$0,022 *$} \\
\hline Sim & $33(22,3)$ & $35(35,7)$ & $1,94(1,10-3,41)$ & \\
\hline \multicolumn{5}{|l|}{ Cardíaca } \\
\hline Não & $138(93,2)$ & $84(82,4)$ & 1 & \multirow{2}{*}{$0,009 *$} \\
\hline Sim & $10(6,8)$ & $18(17,6)$ & $2,96(1,30-6,71)$ & \\
\hline \multicolumn{5}{|l|}{ Prisão de ventre } \\
\hline Não & $143(96,6)$ & $94(92,2)$ & 1 & \multirow{2}{*}{0,129} \\
\hline Sim & $05(3,4)$ & $08(7,8)$ & $2,44(0,77-7,66)$ & \\
\hline \multicolumn{5}{|l|}{ Depressão } \\
\hline Não & $141(95,3)$ & $89(87,3)$ & 1 & \multirow{2}{*}{$0,027 *$} \\
\hline Sim & $07(4,7)$ & $13(12,7)$ & $2,94(1,13-7,66)$ & \\
\hline \multicolumn{5}{|l|}{ Doença nos olhos } \\
\hline Não & $138(93,2)$ & $86(85,1)$ & 1 & \multirow{2}{*}{$0,041 *$} \\
\hline Sim & $10(6,8)$ & $15(14,9)$ & $2,41(1,03-5,60)$ & \\
\hline \multicolumn{5}{|c|}{ Dificuldades auditivas } \\
\hline Não & $143(96,6)$ & $93(91,2)$ & 1 & \multirow{2}{*}{0,076} \\
\hline Sim & $05(3,4)$ & $09(8,8)$ & $2,77(0,90-8,52)$ & \\
\hline \multicolumn{5}{|l|}{ Gastrite } \\
\hline Não & $140(94,6)$ & $92(90,2)$ & 1 & \multirow{2}{*}{0,192} \\
\hline Sim & $08(5,4)$ & $10(9,8)$ & $1,90(0,72-5,00)$ & \\
\hline
\end{tabular}

$\mathrm{Na}$ análise ajustada (Tabela 2), as variáveis que permaneceram associadas ao desfecho foram estado civil, doença cardíaca, depressão e escolaridade. Essas foram consideradas fatores de risco, conforme observado no intervalo de confiança. As mulheres idosas sem companheiro e que estudaram oito ou mais anos apresentaram 2,26 e 1,85 mais chances de desistirem do programa, respectivamente. Em relação à doença cardíaca e a depressão, suas presenças aumentaram as chances em 2,99 e 3,49, respectivamente, de desistirem do programa.

Tabela 2 - Análise ajustada fatores associados a desistência das idosas do programa de atividades física $(n=267)$

\begin{tabular}{lcc}
\hline Variável & OR $(\mathbf{I C ~ 9 5 \%})^{\$}$ & Valor p \\
\hline $\begin{array}{l}\text { Estado Civil } \\
\quad \text { Com companheiro }\end{array}$ & 1 & \\
$\quad \begin{array}{l}\text { Sem companheiro } \\
\text { Cardíaca }\end{array}$ & $2,26(1,27-4,01)$ & 0,005 \\
$\quad$ Não & 1 & \\
$\quad$ Sim & $2,99(1,27-7,03)$ & 0,012 \\
Depressão & 1 & \\
$\quad$ Não & & \\
$\quad$ Sim & $3,49(1,28-9,48)$ & 0,014 \\
Escolaridade & & \\
$\quad$ Até 8 anos & 1 & 0,039 \\
8 anos ou mais & $1,85(1,03-3,31)$ & \\
\hline
\end{tabular}

$\mathrm{OR}=$ Odds Ratio; $\mathrm{IC}=$ intervalo de confiança; $\$=$ ajuste do modelo de $85,4 \%$ (teste de Hosmer-Lemeshow). Fonte: Elaborada pelos autores, 2016. 


\section{Discussão}

O presente estudo apresenta algumas limitações, porque os dados foram obtidos de um banco de dados em que as doenças foram autorrelatadas pelas idosas, sem a comprovação do diagnóstico médico, controle dos níveis de depressão e característica da doença cardíaca.

Os achados do presente estudo apontam que características sociodemográficas como escolaridade, estado civil, presença de doença cardíaca e depressão são fatores que contribuem para a desistência de mulheres idosas de um programa de atividade física universitário.

É evidenciado na literatura que a prática de atividade física regular acarreta benefícios para a saúde do idoso (FRAGOSO et al., 2015; SOARES-MIRANDA et al., 2016; GARFIELD; LLEWELLYN; KUMARI, 2016; KIM et al., 2017), e que a ausência desta prática é relatada como causa de mortalidade para todas as doenças (LEE et al., 2012). Assim, promover a adesão da prática de atividade física e a manutenção desta, tem sido alvo de programas no mundo todo (HARRIS et al., 2015; DONDZILLA et al., 2016). Essa preocupação ocorre devido à prática de atividade física ser considerada um comportamento do movimento humano (GABRIEL; MORROW; WOOLSEY et al., 2012) que é influenciado por determinantes ambientais, sociais, demográficos e individuais (YUSUF et al., 2004), o qual pode ser modificado.

Em relação aos determinantes sociodemográficos, a escolaridade é investigada em alguns estudos (PITANGA et al., 2014; BRONDEEL; PANNIER; CHAIX, 2016; BANN et al., 2016). Na população adulta, indivíduos com níveis de escolaridade mais elevados apresentam três vezes mais chances de praticarem atividade física no tempo livre (PITANGA et al., 2014).Na população idosa, ao investigar as diferenças socioeconômicas nos benefícios de um programa "tradicional" de atividade física e de um programa de educação para saúde, Bann et al. (2016) verificaram que a escolaridade e a renda não foram associadas com os benefícios adquiridos com os dois programas, entretanto, o efeito da intervenção foi maior para os indivíduos com escolaridade mais elevada.

No presente estudo, a escolaridade mais elevada foi associada com a desistência do programa de atividade física. Este indicativo pode significar que pessoas com o perfil supracitado, apresentam mais acesso a oportunidades de atividades físicas no lazer, que pode ser um indicador de desistência de um programa de atividade física vinculado à extensão universitária, com datas e horários mais rígidos e restrição de oferta de atividades físicas.

Ainda em relação aos determinantes sociodemográficos, no presente estudo, as idosas com estado civil sem companheiro apresentaram duas vezes mais chances de desistirem do programa. Esses resultados corroboram a revisão sistemática de Ribeiro et al. (2013), cuja falta de companheiro é um motivo de desistência da prática de atividade física. Isso pode ocorrer, pois a viuvez em idosas mais longevas tem uma relação negativa com a prática de atividade física (LOPES et al., 2016).

Outro ponto importante é evidenciado no estudo de Hawkley, Thisted e Cacioppo (2009), no qual os autores acompanharam, por um período de três anos, 229 homens e mulheres de diferentes etnias, com idade entre 50 e 68 anos,e verificaram que a solidão é um fator de risco para a inatividade física e aumenta a probabilidade de que a atividade física seja interrompida ao longo do tempo. Deste modo, o apoio social recebido pela família e amigos pode contribuir para que o indivíduo mantenha-se praticante de atividade física (MARQUEZ et al., 2015) e está positivamente associado à qualidade de vida relacionada à saúde mental de adultos e idosos (VAN DYCK et al., 2015). Salienta-se que a saúde mental pode ser analisada por diversas perspectivas, incluindo a depressão.

No presente estudo a depressão apresentou-se como um fator associado à desistência, de modo que o grupo que acometido por esta doença manifestou-se, no modelo ajustado, três vezes mais chances de desistir do programa de atividade física. Destaca-se que muitos estudos 
investigaram a adesão e a permanência de forma subjetiva (MOURA; DE SOUZA, 2015; LOPES et al., 2014; SALIN et al., 2014; KRUG; MAZO; LOPES, 2015; LOPES et al., 2016), ocultando os reais motivos que poderiam influenciar nesta desistência. Cabe ressalvar que o referido programa caracteriza-se pelo seu caráter educativo e formativo, o qual visa melhorar a qualidade de vida do idoso cidadão (MAZO et al., 2009). Nesta perspectiva, a metodologia abordada no programa, vai ao encontro da política do envelhecimento ativo, cujo "ativo" refere-se à participação contínua nas questões sociais, econômicas, culturais, espirituais e civis (WHO, 2005). Apesar do importante papel dos programas de atividade física na inserção social das idosas, os resultados obtidos no presente estudo poderão auxiliar no replanejamento de programas de atividade física de modo a suprir as dimensões biológicas e psicossociais do envelhecimento.

Deste modo, é importante ponderar sobre possíveis estratégias para auxiliar no tratamento da depressão para que esta doença não seja uma barreira para a prática de atividades físicas pelas idosas. Neste sentido a meta-análise realizada por Forsman, Schierenbeck e Wahlbeck (2011), com o objetivo de avaliar a eficácia de diferentes intervenções psicossociais para prevenção da depressão em idosos, incluindo o exercício físico, revelaram que as intervenções conjuntas apresentaram efeito estatisticamente significativo na redução de sintomas depressivos.

Outra característica importante que foi associada à desistência do programa de atividade física, foi a presença de doença cardíaca. Na literatura científica, a prática de atividade física é considerada fator de proteção para infarto agudo do miocárdio (YUSUF et al., 2004) e doença coronariana (SOFI et al., 2008; LEE et al., 2012). Por outro lado, revisões sistemáticas demonstram que o comportamento sedentário é fator de risco para doenças cardiovasculares (GROTVED; HU, 2011; FORD; CASPERSEN, 2012).

Entretanto, a doença cardíaca foi associada à desistência do programa. Esse fato pode ocorrer, pois o programa de atividades físicas oferecido não é uma proposta específica para reabilitação cardíaca, com caráter de tratamento, e, sim, uma proposta de promoção de saúde baseada na política do envelhecimento ativo. O que ocorre, é que na mesma instituição existe um programa de reabilitação cardíaca por meio da prática de atividade, que supre essa necessidade específica de atendimento.

\section{Conclusão}

Esse estudo acrescenta novas informações à comunidade científica ao apresentar variáveis sociodemográficas (escolaridade e estado civil), presença de depressão e de doença cardíaca, como fatores associados à desistência de um programa de extensão universitário de atividades físicas. Os resultados demonstram que estratégias devem ser adotadas, objetivando a manutenção regular desta prática. Para tanto, é necessário um replanejamento sistemático dos objetivos e ações do programa de modo a efetivar a participação dessas idosas. Dessa forma, os achados deste estudo podem melhorar a intervenção no programa universitário envolvido, e, também serve como subsidio para a implementação de novos programas de promoção da saúde por meio da atividade física.

Considerando que os programas de extensão universitários articulam pesquisa e ensino, torna-se pertinente levar estas informações às disciplinas desenvolvidas no curso de graduação para contribuir na formação e qualificação profissional de ingressantes no mercado de trabalho. Deste modo, a Universidade cumpre seu papel na produção de mudanças na sociedade. 


\section{Referências}

BANN, D. et al. Life Study investigators. Socioeconomic differences in the benefits of structured physical activity compared with health education on the prevention of major mobility disability in older adults: the LIFE study. J Epidemiol Community Health, v. 70, n. 9, p. 930-3, 2016.

BAUMAN, A. E. et al. Lancet Physical Activity Series Working Group. Correlates of physical activity: why are some people physically active and others not? Lancet, v. 380, n. 9838, p. 258-71, 2012.

BIELEMANN, R. M. et al. Burden of physical inactivity and hospitalization costs due to chronic diseases. Rev Saúde Pública, v. 49, p. 1-8, 2015.

BLOCH, K. V.; COUTINHO, E. S. F. Fundamentos da Pesquisa Epidemiológica. In: MEDRONHO, R. A. Epidemiologia. 2.ed. São Paulo: Atheneu, 2009.

BRONDEEL, R.; PANNIER, B.; CHAIX, B. Associations of socioeconomic status with transport-related physical activity: combining a household travel survey and accelerometer data using random forests. J Transp Health, v.3, p. 287-296, 2016.

DONDZILLA, C. J.et al. Translating exercise interventions to an in-home setting for seniors: preliminary impact on physical activity and function. Aging Clin Exp Res, v. 28, p. 1227$1235,2016$.

FORD, E. S.; CASPERSEN, C. J. Sedentary behaviour and cardiovascular disease: a review of prospective studies. Int J Epidemiol, v. 41, n. 5, p. 1338-1353, 2012.

FORSMAN, A. K.; SCHIERENBECK, I.; WAHLBECK, K. Psychosocial interventions for the prevention of depression in older adults: systematic review and meta-analysis. J Aging Health, v. 23, n. 3, p. 387-416, 2011.

FRAGOSO, C. A. V. et al. Effect of Structured Physical Activity on Sleep-Wake Behaviors in Sedentary Elderly Adults with Mobility Limitations. J Am Geriatr Soc, v. 63, p. 13811390, 2015.

GABRIEL, K. K. P.; MORROW, J. R. J. R.; WOOLSEY, A.L. Framework for Physical Activity as a Complex and Multidimensional Behavior. J Phys Act Health, v. 9, n. 1, p. 11-18, 2012.

GARFIELD, V.; LLEWELLYN, C. H.; KUMARI, M. The relationship between physical activity, sleep duration and depressive symptoms in older adults: The English Longitudinal Study of Ageing (ELSA). Prev Med Rep, v. 4, p. 512-516, 2016.

GROTVED, A.; HU, F. B. Television Viewing and Risk of Type 2 Diabetes, Cardiovascular Disease, and All-Cause Mortality A Meta-analysis. JAMA, v. 305, n. 23, p. 2448-2455, 2011.

HARRIS, T.et al. A Primary Care Nurse-Delivered Walking Intervention in Older Adults: PACE (Pedometer Accelerometer Consultation Evaluation)-Lift Cluster Randomised Controlled Trial. Plos Med, v. 12, n. 2, 2015.

HAWKLEY, L. C.; THISTED, R. A.; CACIOPPO, J. T. Loneliness Predicts Reduced Physical Activity: Cross-Sectional \& Longitudinal Analyses. Health Psychol, v. 28, n. 3, p. 354363, 2009. 
IBGE- Instituto Brasileiro de Geografia E Estatística. Síntese de Indicadores Sociais - Uma análise das condições de vida da população brasileira 2013. Rio de Janeiro: Ministério do Planejamento, Orçamento e Gestão. Diretoria de Pesquisas. Coordenação de População e Indicadores Sociais. Estudos e Pesquisas, Informação Demográfica e Socioeconômica, 2013.

IBGE - Instituto Brasileiro de Geografia e Estatística. Síntese de indicadores sociais: uma análise das condições de vida da população brasileira. Rio de Janeiro: Ministério do Planejamento, Orçamento e Gestão, 2015.

KIM, E.S. et al. Maintaining Healthy Behavior: a Prospective Study of Psychological WellBeing and Physical Activity. Ann Behav Med, v. 51, n. 3, p. 337-347, 2017.

KRUG, R. R.; MAZO, G. Z.; LOPES, M. A. Barreiras e facilitadores para a prática da atividade física de longevas inativas fisicamente. Rev Bras Med Esporte, v. 21, n. 1, p. 57-64, 2015.

LEE, I. M. et al. Lancet Physical Activity Series Working Group. Effect of physical inactivity on major non-communicable diseases worldwide: an analysis of burden of disease and life expectancy. Lancet, v. 380, n. 9838, p. 2019-29, 2012.

LOPES, A. L. et al. Motivos de ingresso e permanência em um programa de atividades aquáticas: um estudo longitudinal. Rev. Educ. Fís/UEM, v. 25, n. 1, p. 21-32, 2014.

LOPES, M. A. et al. Barriers that influence the non-adoption of physical activity practice by oldest old people. Rev. Bras. Ciênc. Esporte, v. 38, n. 1, p. 76-83, 2016.

MARQUEZ, B. et al. Social support and physical activity change in Latinas: Results from the Seamos Saludables trial. J Racial Ethn Health Disparities, v. 2, n. 3, p. 351-357, 2015.

MAZO, G. Z. et al. Do diagnóstico à ação: Grupo de Estudos da Idoso: Alternativa para a promoção do envelhecimento ativo. Rev Bras Ativ Fis Saúde, v. 14, n. 1, p. 65-70, 2009.

MIKKELSEN, S. S. et al. A cohort study of leisure time physical activity and depression. Prev Med, v. 51, n. 6, p. 471-475, 2010.

MOURA, A. O. D.; DE SOUZA, L. K. Grupos de convivência para idosos: participantes, egressos e desinteressados. Estud. Pesqui. Psicol., v. 15, n. 3, p. 1045-1060, 2015.

NELSON, M. E. et al. Physical activity and public health in older adults: recommendation from the American College of Sports Medicine and the American Heart Association. Circulation, v. 116, n. 9, p. 1094-2007, 2007.

PITANGA, F. G. et al. Prevalência e fatores sociodemográficos e ambientais associados à atividade física no tempo livre e no deslocamento em adultos. Motricidade, v. 10, n. 1, p. 3$13,2014$.

RIBEIRO, L. B. et al. Motives of adherence and dropout of elderly to physical activity practice. Rev Bras Promoc Saude, v. 26, n. 4, p. 575-582, 2013.

SALIN, M. S. et al. Golden Age Gym: reasons for entry, permanence and satisfaction among participating older adults. Rev Bras Cineantropom Desempenho Hum, v. 16, n. 2, p. 152160, 2014. 
SOARES-MIRANDA, L. et al. Physical Activity and Risk of Coronary Heart Disease and Stroke in Older Adults: The Cardiovascular Health Study. Circulation, v. 133, n. 2, p. 147$155,2016$.

SOFI, F. et al. Physical activity during leisure time and primary prevention of coronary heart disease: an updated meta-analysis of cohort studies. Eur J Cardiovasc Prev Rehabil, v. 15, n. 3, p. 247-257, 2008.

VAN DYCK, D. et al. Relationship of the Perceived Social and Physical Environment with Mental Health Related Quality of Life in Middle-Aged and Older Adults: Mediating Effects of Physical Activity. Plos One, v. 10, n. 3, 2015.

WALSTON, J. et al. Research agenda for frailty in older adults: toward a better understanding of physiology and etiology: summary from the American Geriatrics Society/ National Institute on Aging Research Conference on Frailty in Older Adults. J Am Geriatr Soc, v. 54, n. 15, p. 991-1001, 2006.

WHO - World Health Organization. Envelhecimento ativo: uma política de saúde. Brasília: Organização Pan-Americana da Saúde, 2005.

YUSUF, S. et al. INTERHEART Study Investigators. Effect of potentially modifiable risk factors associated with myocardial infarction in 52 countries (the INTERHEART study): case-control study. Lancet, v. 364, n. 9438, p. 937-952, 2004.

Recebido em: 21/11/2017

Revisado em: 12/09/2018

Aprovado em: 14/11/2018

Endereço para correspondência:

enaianemenezes@gmail.com

Enaiane Cristina Menezes

Universidade do Estado de Santa Catarina

Av. Me. Benvenuta, 2007

88035-901 Itacorubi, Florianópolis - SC, Brasil 